\section{Tailored to} implant patients

The implant kit from Molar contains a comprehensive range of products, specifically for use by the implant patient. The kit includes a special care toothbrush and a TePe select extra soft toothbrush for general oral hygiene. The implant and interspace brush can be used for specific cleaning around implant abutment posts. Sample packs of the TePe interdental brushes and proximal brush are included in the kit, as is a toothbrush

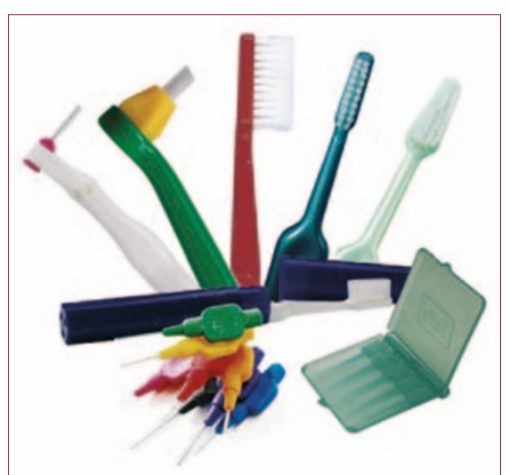

travel case which can be used for any of the TePe toothbrushes. Also included are plastic interdental sticks and proxi-floss.

The Implant kit is available from Molar for $£ 9.90$ each with a minimum order of three packs.

\section{Avoiding cross infection}

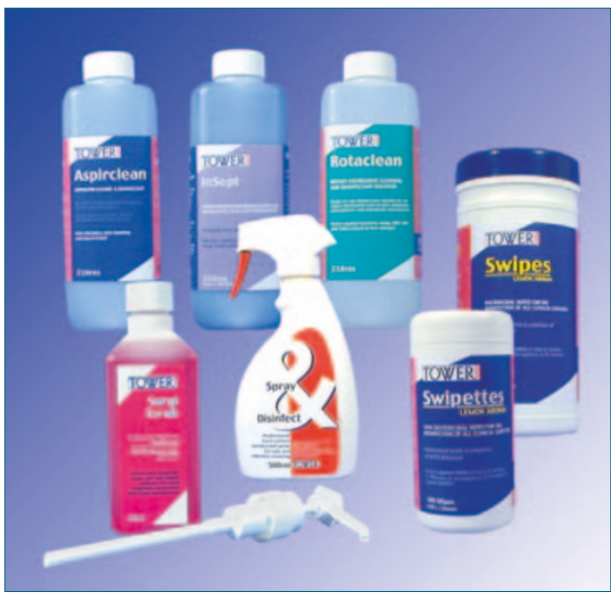

Tower Dental's professional infection control products range from hard surface cleaners to hand scrubs, all helping to ensure that the dental surgery is kept surgically clean. Aspirclean is a new aspirator cleaner and disinfectant which can be used on wet and dry line systems as well as semi-wet/dry line systems. Non-abrasive, non-foaming and bactericidal, Aspirclean is easy to use and measure. It has a high alkaline content to neutralise acid-based substances and is aldehyde, chlorine and phenol free.

\section{Association contacts}

\section{BRITISH ASSOCIATION OF DENTAL NURSES}

Pam Swain (Chief Executive), PO Box 4, Room 200, Hillhouse International Business Centre, Thornton-Cleveleys, FY5 4QD Tel: $\quad 01253338360$ Email: admin@badn.org.uk Website: www.badn.org.uk

\section{BRITISH ASSOCIATION OF DENTAL THERAPISTS}

Jane Lewis (Secretary), c/o 92 Mayfield Drive, Caversham, Reading, Berkshire, RG4 5JT

Tel: $\quad 01189482651$

Email: badt@freeuk.com

Website: www.badt.org.uk

\section{BRITISH DENTAL}

\section{HYGIENISTS' ASSOCIATION}

Ann Craddock (Administrator),

Mobbs Miller House, Ardington Rd,

Northampton, NN1 5LP

Tel: $\quad 08702430752$

Email: enquiries@bdha.org.uk

Website: www.bdha.org.uk

\section{BRITISH DENTAL PRACTICE} MANAGERS' ASSOCIATION

Bridget Crump (Chairman), Osprey House, Primett Road, Stevenage, Herts, SG1 3EE Tel: $\quad 08708400381$

Email: info@bdpma.org.uk Website: www.bdpma.org.uk

\section{DENTAL TECHNICIANS' ASSOCIATION}

Sue Adams (Registrar),

PO Box 6520, Northampton, NN3 9ZX

Tel: $\quad 08702430753$

Email: sueadams@dta-uk.org

Website: www.dta-uk.org

\section{ORTHODONTIC NATIONAL GROUP OF DENTAL NURSES}

Maureen Dickinson (Chairman),

Department of Orthodontics,

Scarborough General Hospital, Woodlands

Drive, Scarborough, YO12 6QL

Tel: $\quad 01723342113$

Email: maureen.dickinson @acute.sney.nhs.uk

Website: www.orthodontic-ong.co.uk

\author{
DENTAL LABORATORIES \\ ASSOCIATION \\ Michael Egerton (Chairman), \\ 44-46 Wollaton Road, \\ Beeston, Nottingham \\ NG9 2NR \\ Tel: $\quad 08709914525$ \\ Email: enquiries@dla.org.uk \\ Website: www.dla.org.uk

\section{ORTHODONTIC} \\ TECHNICIANS' ASSOCIATION \\ Paul Mallett (Secretary), \\ The Maxillofacial Laboratory, \\ Royal Lancaster Infirmary, \\ Ashton Road, \\ Lancaster, Lancs \\ LA1 4RP \\ Website: www.orthota.co.uk

\section{BRITISH DENTAL} \\ RECEPTIONISTS' ASSOCIATION \\ Gemma Bowen, \\ 24 Farnworth Grove, \\ Castle Bromwich, \\ Birmingham \\ B36 9JA \\ Tel: $\quad 08700801924$ \\ Email: gemma@bdra.co.uk \\ Website: www.bdra.co.uk
}

